https://doi.org/10.46344/JBINO.2021.v10i04.13

\title{
INFANTS IMMUNIZATION: CHALLENGES OF OTHER VACCINES DUE TO COVID-19 PANDEMIC
}

\author{
*Emmanuel Ifeanyi Obeagu1, Quratulain Babar'2, C.C.N. Vincent ${ }^{3}$ and Confidence Ogechi Anyanwu 4 \\ 'Department of Medical Laboratory science, Imo State University, Owerri, Nigeria \\ 2Department of Biochemistry, Government College University, Faisalabad, Pakistan \\ 3Department of Nursing Science, Imo State University, Owerri, Nigeria. \\ ${ }^{4}$ Department of Medical Laboratory Science, Madonna University Nigeria, Elele Campus, Rivers State, Nigeria.
}

Email: emmanuelobeagu@yhoo.com

\begin{abstract}
Severe acute respiratory syndrome coronavirus 2 related diseases (COVID-19) is now responsible for one of the most challenging and concerning pandemics. By August 2020, there were almost 20 million confirmed cases worldwide and well over half-million deaths. Since there is still no effective treatment or vaccine, non-pharmaceutical interventions have been implemented in an attempt to contain the spread of the virus. During times of quarantine, immunization practices in all age groups, especially routine childhood vaccines, have also been interrupted, delayed, re-organized, or completely suspended. Numerous highincome as well as low- and middle-income countries are now experiencing a rapid decline in childhood immunization coverage rates. We will, inevitably, see serious consequences related to suboptimal control of vaccine-preventable diseases (VPDs) in infant's concurrent with or following the pandemic. Routine pediatric immunizations of individual infants at clinics, mass vaccination campaigns, and surveillance for VPDs must continue as much as possible during pandemic.
\end{abstract}

Keyword: vaccine, infants, immunity, covid-19 


\section{INTRODUCTION}

Unlike many infectious diseases, such as endemic malaria and common flu where infants are known to have the highest mortality rates and to drive transmission in households and communities it appears as it could be that SARS-CoV-2 just does not translate into severe disease as frequently in infants, specifically for young children, below 10 years of age (1). Moreover, infected infants suffer milder symptoms of COVID-19, with much lower case-fatality rates (CFR), and recover quickly from the infection (2). In an initial assessment from Wuhan, China, among 50 infants identified with COVID-19, the severity varied between asymptomatic and mild in $96 \%$ of the patients (3). While diagnostic findings were similar to those of adults, fewer infants developed severe pneumonia. Neonates, on the other hand, have developed symptomatic and more severe COVID-19 (4).

1 The Immunological Basis for Potential Effect of Childhood Vaccines in Disease Expression

In many countries, infants are routinely vaccinated against a number of bacterial and viral diseases. Vaccines may have non-specific physiologic effects when they alter the immune response to unrelated organisms, called heterologous immunity. The non-specific effects of vaccines are usually more pronounced in girls and appear to be maximal in the first 6 months of life when passed maternal immunity is further supplemented by newly introduced vaccines, starting at 2 months. There are several theories as to why heterologous immunity may occur (5).
Salman and Salem suggest that crossimmunogenicity of childhood vaccines for multiple viruses could potentially be a reason for the relatively milder infection and severity of COVID-19 among infants (1). Most routine viral vaccines are either inactivated or killed viruses that stimulate $T$ Helper 1 cells (CD4+) to secrete many different types of cytokines as interferon gamma, interleukin-2 (IL-2), and IL-12, improving the cytotoxicity of natural killer cells to recognize and destroy cells infected with new cross-reactive viruses. For example, warts that are caused by human papilloma virus (HPV) could be ameliorated using intralesional MMR vaccine (6).

Furthermore, neutralizing antibodies produced against the foregoing vaccinepreventable microbes might cross-react with the antigenic epitopes of the spike (S) and nucleocapsid (N) proteins and prevent COVID-19 in infants (7). An investigation of this hypothesis, using the BLAST search tool, showed no significant sequence similarity between these proteins and those in the childhood vaccinepreventable microbes, inferring that memory T-cells, rather than vaccine neutralizing antibodies, may be involved in the protection of infants against COVID-19 owing to them having a larger number of naive T-cells that can be programmed to protect them against the disease (8).

Potentially, the low immunity in infants that doesn't exaggerate the immune response against the virus as in the case of adults, could explain the lesser severity of SARSCoV-2 in this age group. Infants have less 
adults-like memory cells specific to other circulating coronaviruses and therefore, are less capable to mount a devastating and vigorous cell-mediated attack on alveoli and interstitial tissue of the lung upon new infection (9).

\subsection{The Bacille Calmette-Guérin (BCG) Vaccine}

O'Neil and Netea suggest that induction of trained immunity by BCG vaccine could provide protection against COVID-19, and the use of oral polio vaccine and new recombinant BCG-based vaccine VPM 1002 may be some of the approaches to induce resistance to SARS-CoV-2 (10). The authors hypothesize that induction of trained immunity is at least partly the mechanism through which BCG vaccination induces its beneficial effects and might protect against SARS-CoV-2. A retrospective study compared countries that do not have BCG vaccination policies (Italy, USA, Lebanon, the Netherlands, and Belgium), to countries that have such policies (11). The results showed that while middle-high and high-income countries with current universal BCG policies had 0.78 COVID-19 deaths per million, those without such policies had 16.39 COVID-19 deaths per million people and the difference was statistically significant. In countries, such as Italy, where BCG vaccine was never given, the mortality rate was significantly higher compared to Japan where BCG vaccination has been implemented since 1947. In countries, such as Iran, with BCG vaccination starting in 1984, mortality was higher since today's elderly population did not receive the vaccination.
COVID-19 mortality rates in New York, Illinois, Alabama and Florida states without BCG-vaccination policies in the US, were significantly higher than locations with BCG-vaccine policies, namely Pernambuco, Rio de Janeiro, and Sao Paulo in Brazil, or Mexico State and Mexico City in Mexico.

\subsection{The Measles, Mumps, and Rubella (MMR) Vaccine}

Two potential mechanisms for higher COVID-19 cases per population ratio and higher death rate in Italy (no MMR vaccine) compared to China: (1) by generating bystander immunity the measles vaccine increases ability of immune system to combat non-measles pathogens, including coronaviruses, and (2) due to shared structural similarities between measles and coronavirus the cross-reactivity and immunity between the measles vaccine and coronavirus leads to partial protection against COVID-19 (12). Franklin et al., identified that the macro domains of SARS-COV-2 and rubella virus and the MMR vaccine, share $29 \%$ amino acid sequence identity (13). This finding suggests the viruses possess the same protein fold. Patients with high illness severity had high levels of rubella lgG $(161.9+147.6 \mathrm{IU} / \mathrm{ml})$ compared to patients with a moderate severity of disease $(74.5+$ $57.7 \mathrm{IU} / \mathrm{ml})$. The authors suggest the MMR vaccine could result in potentially reduced severe outcomes with COVID-19. In their commentary, Fidel and Noverr support the use of live attenuated MMR vaccine as a preventive measure against the pathological inflammation and sepsis associated with COVID-19 infection (1). 


\subsection{The Hepatitis-A (HEP-A) Vaccine}

Sarialioglu et al., reported on the differences in the rate in which COVID-19 had affected some countries such as China, US, Italy, Spain, France, England, the Netherlands, and Belgium more severely than some others such as India, Pakistan, countries of the African continent, and South America which had lower rates of infection and mortality at the time of their study (15). The authors hypothesize that routine vaccination for hepatitis A virus (HAV) causing high seroprevalence among populations in countries in the low COVID-19 prevalence group, while it is rather low in the industrialized countries. The authors conclude that immune response caused by the hepatitis A vaccine may be protective against COVID-19 infection by a possible adaptive immune cross-reaction. Patients with asymptomatic COVID-19 disease could indirectly indicate those with protection from HAV seropositivity. The HEPA vaccine may help to keep the COVID-19 infection at mucosal colonization levels and prevent lower respiratory tract involvement and fatality (1).

2 Scenario of Routine immunizations during the COVID-19 pandemic

COVID 19 is disrupting life-saving immunization services around the world, putting millions of infants in industrialized as well as in low- and middle-income countries - at risk of vaccine-preventable diseases. Because of the risk of infection and the need to maintain physical distance during the early stages of the COVID-19 pandemic, many countries have temporarily and reasonably suspended preventive mass vaccination campaigns against diseases such as measles, polio, diphtheria, pertussis, polio, tetanus, meningitis (16). Causes of the delayed/interrupted immunizations are due to parents' fears, restrictions of movement/lockdown policies, changing priorities for COVID-19 among health-care personnel, and logistics delivery issues (i.e., vaccine transport delays). The World Health Organization (WHO), UNICEF, and GAVI, the Vaccine Alliance have reported that routine immunization programs have been substantially disrupted in at least 68 countries, affecting around 80 million infants (15). A further 24 million people are at risk of losing out on vaccines including measles, polio, rotavirus, meningitis, rubella, and human papillomavirus in GAVIsupported low-income countries (16). Vaccines are of critical importance in these areas, which also have limited access to health services and treatments. Due to the risk of inadvertently contributing to the spread COVID-19, global health organizations have not been able to continue to support mass vaccination campaigns in some countries (17).

\section{Global Suspension of vaccine service}

Of the 129 countries in which data were available, more than half reported moderate to severe disruptions, or total suspension of vaccination services during March - April 2020. There was a full cessation of routine immunizations from April 1-15, 2020 in Vietnam. In India, routine immunizations were disrupted due to health-care workers being re-organized in response to the pandemic. In Pakistan, polio catch-up immunization campaigns

\section{July Edition |www.jbino.com | Innovative Association}


were postponed until June 1st, 2020(18). A number of countries postponed immunization campaigns in the first 5 months of the pandemic, including: measles or measles-containing vaccines in 27 countries, inactivated polio vaccine in 7 countries, bivalent, or monovalent oral poliovirus vaccine in 39 countries, meningococcal conjugated $A$ vaccine in 2 countries, yellow fever vaccine in 4 countries, typhoid vaccine in 2 countries, oral cholera vaccine in 5 countries, and Td (tetanus-diphtheria) vaccine in 7 countries (19). While several mass immunization campaigns have been stopped to prevent the virus spreading, the results have been alarming. Diphtheria has recently resurged in countries like Venezuela, Pakistan, Nepal, Bangladesh, and Yemen where conflict and/or population displacement have seriously affected public health systems (20). Cholera is now present in Bangladesh, Cameroon, Mozambique, South Sudan, and Yemen (15).

\section{Outrage of polio}

Polio is an important concern, and suspending polio immunizations is a particularly relevant issue where the virus is likely to spread (e.g., Afghanistan and Pakistan) due to active circulation. The risk of polio spreading to other countries is real, and could negatively affect global polio eradication efforts (21).

The Global Polio Eradication Initiative recommended suspending polio vaccination campaigns until the second half of 2020 (22). As a result of the coronavirus pandemic, 46 poliovirus immunization campaigns in 38 countries, mostly in Africa have been suspended.
During this time, a mutated vaccinederived strain of poliovirus has been reported in $>30$ countries (23). Following the suspension of these immunization activities, there has been a new polio outbreak in Niger (24). In Pakistan and Afghanistan, wild poliovirus Type 1 have been reported, and cases of Type 2 poliovirus, mutated from the oral vaccine, have appeared in Chad, Ethiopia, Ghana, and Pakistan (25).

\section{Measles outrage}

Measles is a disease that can cause serious morbidity and has a casefatality rate of $0.2 \%$, it is more highly contagious (basic reproduction rate; RO as 12-16) than COVID-19 (26).During the pandemic, 37 countries (home to 117 million unvaccinated imfants) suspended scheduled measles campaigns, and more will be postponed (27). Before the pandemic, and despite the availability of an effective vaccine, the highly contagious measles virus continued to spread around the globe. In 2018, cases surged to an estimated 10 million worldwide, with 140,000 deaths, a $58 \%$ increase since 2016 (28). Measles outbreaks have been reported in diverse settings, including Madagascar and Ukraine (29). In 2019, measles outbreaks infected more than 6,500 infants in the Democratic Republic of the Congo where one-dose measles vaccine coverage was 57\% (30). In 2019, in many countries faced vaccination coverage rates below the minimum needed for herd immunity; this posed a serious risk to infants who were not vaccinated and indirectly threatened immunocompromised infants and others 
who could not be vaccinated for other medical contraindications. Eighteen countries reported measles outbreak due to suspended measles campaigns. By 2020, 178 million people are at risk of skipping measles vaccines, according to the Measles and Rubella Initiative, even as measles flares across the globe, including in Afghanistan, Brazil, Cambodia, the Central African Republic, Iraq, Kazakhstan, Nepal, Nigeria, and Uzbekistan (31).

Measles weaken the immune system for months or years, causing immune amnesia that leaves infants susceptible to other infections (32).

\section{Potential effects of existing vaccines on COVID-19 pandemic}

A majority of countries include conjugated pneumococcal vaccines in their national childhood immunization programs and recommend conjugated and/or polysaccharide vaccines for high-risk groups and elderly people. Routine immunizations that include pneumococcal vaccines for infants and high-risk adults should continue according to previously developed schedules, to reduce the need for hospital admissions for vaccinepreventable diseases. High influenza vaccine coverage would reduce influenzarelated mortality while also helping to preserve the capacity and function of the health system during circulation of both influenza and COVID-19 viruses. The goal should be to attain high influenza vaccine coverage, including near-universal coverage among healthcare personnel and other high-risk groups for COVID-19 (33).
7 Routine immunizations in infants and adults with suspected or confirmed COVID-19 infections

There is currently no evidence that vaccination would increase the risk of a child becoming infected with COVID-19, or affect the course of the disease in a child who has been inadvertently vaccinated during the asymptomatic phase or incubation period (34). In cases of mothers with a laboratory-confirmed diagnosis of COVID-19, asymptomatic newborns could be vaccinated according to local recommendations with BCG and hepatitis $B$ vaccine. If the newborn presents COVID19 symptoms, the BCG vaccine should be postponed and the hepatitis $B$ vaccine might be administered within the first 24 hours of life (35). Here is no clear guidance on the administration of routine vaccines in infants with COVID-19, or those who have tested positive for the virus but who have no symptoms or known contact. Individuals with signs of acute respiratory infection, including mild symptoms such as sore throat or runny nose, may postpone regular immunization until they have recovered during the COVID-19 pandemic. The presence of fever may cause confusion concerning vaccine side effects or the progression of the illness; therefore, vaccines can be postponed until illness resolution, in cases of acute febrile illness (34). The CDC recommends deferring vaccinations of anyone infected with SARS-CoV-2, regardless of symptoms, until they meet the criteria to discontinue isolation. It is also prudent to delay the administration of any live vaccines among COVID-19 patients who have been treated 
with immunoglobulin or plasma, according to routine live vaccine use protocols.

\section{Immunization measures by WHO during the COVID-19 pandemic}

The WHO recommends that all routine vaccinations be administered as scheduled, even during the COVID-19 pandemic. Routine immunization sessions should continue, using special measures and precautions, to the extent possible and as permitted within the local COVID19 response context. This includes routine immunization for infants, children, adolescents, pregnant women, high-risk groups, and adult healthcare providers (36). Any interrupted immunization services should be resumed and catch-up vaccinations offered as quickly as possible (34). New vaccines should be delayed from introduction to national immunization schedules. It should be communicated clearly to the community and healthcare professionals alike the rationale for the inclusion of immunizations among the priority health services provided during the COVID-19 pandemic. The risks from VPDs and the benefits of vaccination should also be reiterated (37).

\section{Myths about vaccination}

Vaccine hesitancy/refusal has been one of the most important health problems in recent years, and during the COVIDI9 period, the most radical groups have continued to share false and nonevidence-based opinions with the community (38). Many of these theories posed during the pandemic have the risk of increasing future vaccine refusals. One of the most important observations during the period has been that the speed of transmission of false information about the disease is much faster and more dangerous than the speed of the spread of the virus (39).

Reduced access to critical health-care facilities and medications is known to establish circumstances in which patients use substandard drugs and/or dosages. This could lead to increased morbidity and mortality caused by human immunodeficiency virus infection, tuberculosis, and malaria, as well as leading to the rise and spread of drugresistant diseases (40). These populations need protection not only from the pandemic, but also from the consequences of non-pharmaceutical interventions. In addition to low- and middle-income countries, other vulnerable populations for the pandemic and mitigation strategies consequences are refugees and migrants. The pandemic has had an enormous effect on mobility, border and migration management, and on global migrant populations' health, social, and economic situation. There now needs to be an immediate concerted effort to link these communities with national and global responses to COVID-19 (41). During the pandemic, immunization program for refugees and immigrants should not be neglected.

\section{Causes of outbreaks}

In many countries, vaccine hesitancy and refusal have become an important problem in recent years.30 Due to school closures, serious immunization disruptions may occur in countries where routine immunizations have been delivered in school settings. According to UNESCO 
data from April 15, 2020, 91\% of the world's school-age children have stopped their education due to the pandemic (42). If children are left unvaccinated, case numbers could quickly surge following a drop in the optimal population immunity and the suspension of these vaccination programs. Parental fears about potentially exposing their infants to COVID-19 during routine follow-up visits may have led to the declines observed (43).

11 Cross talk of previous vaccination immunity and covid-19 infection

Previous studies showed that live vaccines (i.e., BCG, oral polio vaccine, measles) induce heterologous protection against infections, likely by inducing interferon and with a long-term boosting of innate immune immunity mechanisms (44). Measles and smallpox vaccines have also been associated with pronounced nonspecific protective effects against infectious diseases. Both poliovirus and coronavirus are positive-strand RNA viruses; thus, specific innate immunity mechanisms are likely to induce and affect these viruses.

\section{Conclusion and future perspectives}

Vaccination and immunization is responsible for protection from severity of disease. COVID-19 is creating hurdles in the field of immunization, so we should take proper measures regarding this. As no proper vaccine for covid-19 for children and infants is launched that's why BCG, hepatitis and polio vaccines have proved beneficial to create protective antibodies against corona virus. Through these useful information we can make strategies for formation of COVID 19 vaccination in future by using previous FDA approved Infants vaccines.

\section{Reference}

1. Beric-Stojsic, B., Kalabalik-Hoganson, J., Rizzolo, D., \& Roy, S. (2020). Childhood immunization and COVID-19: an early narrative review. Frontiers in Public Health, 8.

2. Singh $T$, Hesto $S M$, Langel SN, Blasi M, Hurst $J H$, Fouda GG, et al. Lessons from COVID19 in children: key hypotheses to guide preventative and therapeutic strategies. Clin Infect Dis. (2020) ciaa547. doi: 10.1093/cid/ciaa547

3. Ma H, Hu J, Tian J, Zhou X, Li H, Laws M et al. Visualizing the novel coronavirus (COVID-19) in children: what we learn from patients at wuhan children's hospital. SSRN Electron J. (2020). doi: 10.2139/ssrn.3556676

4. Ludvigsson JF. Systematic review of COVID19 in children shows milder cases and a better prognosis than adults. Acta Paediatrica. (2020) 109:1088-95. doi: 10.1111 /apa.15270

5. Salman S, Salem M. Routine childhood immunization may protect against COVID19. Med Hypotheses. (2020). doi: 10.1016/j.mehy.2020.109689. [Epub ahead of print].

6. Shann F. The non-specific effects of vaccines. Arch Dis Childhood. (2010) 95:662-7. doi: 10.1136/archdischild-2015310282

7. Sabir DK, Sidiq KR, Ali SM. Current speculations on the low incidence rate of the COVID-19 among children. Int. J. School. Health. (2020) 7:61-2. doi: 10.30476/intjsh.2020.85997.1066

8. Ahmadpoor PL, Rostaing L. Why the immune system fails to mount an adaptive 2021 July Edition | www.jbino.com | Innovative Association 
immune response to a COVID-19 infection. Transpl Int. (2020) 33:824-5. doi: $10.1111 /$ tri. 13611

9. Abdulamir AS, Hafidh RR. The possible immunological pathways for the variable immunopathogenesis of covid-19 infections among healthy adults, elderly and children. Electron J Gen Med. (2020) 17:em202. doi: 10.29333/ejgm/7850

10. O'Neill LA, Netea MG. BCG-induced trained immunity: can it offer protection against COVID-19? Nat Rev Immunol. (2020) 20:335-7. doi: 10.1038/s41577-020-0337-y

11. Miller A, Reandelar MJ, Fasciglione K, Roumenova V, Li Y, Otazu GH. Correlation between universal BCG vaccination policy and reduced morbidity and mortality for COVID-19: an = epidemiological study. medRxiv [Preprint]. (2020). doi: 10.1101/2020.03.24.20042937

12. Saad M, Elsalamony R. Measles vaccines may provide partial protection against COVID-19. Int J Cancer Biomed Res. (2020) 5:14-19. doi: 10.21608/jcbr.2020.26765.1024

13. Franklin R, Young A, Neumann B, Fernandez R, Joannides A, Reyahi A, et al. Homologous protein domains in SARS-CoV2 and measles, mumps and rubella viruses: Preliminary evidence that MMR vaccine might provide protection against COVID19. medRxiv [Preprint]. (2020) doi: 10.1101/2020.04.10.20053207

14. Sarialioglu F, Apak FBB, Haberal M. Can hepatitis a vaccine provide protection against covid-19? Exp Clin Transpl. (2020) 2:141-3. doi: 10.6002/ect.2020.0109

15. Dinleyici, E. C., Borrow, R., Safadi, M. A. P., van Damme, P., \& Munoz, F. M. (2021). Vaccines and routine immunization strategies during the COVID-19 pandemic. Human vaccines immunotherapeutics, 17(2), 400-407.

16. [accessed 2020 Jun 29]. https://www.who.int/news-room/detail/ 2205-2020-at-least-80-million-children-underone-at-risk-of- diseases-such-as-diphtheriameasles-and-polio-as-covid-19- disruptsroutine-vaccination-efforts-warn-gavi-whoand-unicef.

17. Niger reports new polio outbreak. [accessed 2020 Jun 29]. https:// www.who.int/immunization/diseases/meas| es/statement_missing

measles_vaccines_covid-19/en/. 82023

18. Nelson R. COVID-19 disrupts vaccine delivery. Lancet Infect Dis. Apr 172020. doi:10.1016/S1473-3099(20)30304-2.

19. [accessed 2020 Jun 29]. https://www.who.int/news-room/detail/ 2205-2020-at-least-80-million-children-underone-at-risk-of- diseases-such-as-diphtheriameasles-and-polio-as-covid-19- disruptsroutine-vaccination-efforts-warn-gavi-whoand-unicef.

20. Hoffman J, Maclean $R$ Slowing the Coronavirus is speeding the spread of other diseases. New York Times. 22 Jun 2020. https:// www.nytimes.com/2020/06/14/health/coro navirus-vaccines- measles.html. [accessed 2020 Jun 29]

21. Education: From disruption to recovery. [accessed 2020 Jun 29]. http://polioeradication.org/poliotoday/polio-now/this-week/.

22. Roberts L. Global polio eradication falters in the final stretch. Science. 2020 Jan 3;367(6473):14-15. doi:10.1126/science.367.6473.14. 
23. [accessed

2020

Jun

29].

https://www.who.int/news-room/detail/ 22-

05-2020-at-least-80-million-children-under-

one-at-risk-of- diseases-such-as-diphtheriameasles-and-polio-as-covid-19- disruptsroutine-vaccination-efforts-warn-gavi-whoand-unicef.

24. [accessed 2020

Jun

29]. https://www.afro.who.int/news/nigerreports-new-polio-outbreak.

25. [accessed 2020 Jun 29]. http://polioeradication.org/polio-today /polio-now/this-week/. 32226

26. Grasselli G, Zangrillo A, Zanella A, Antonelli M, Cabrini L, Castelli A, Cereda D, Coluccello A, Foti G, Fumagalli $R$, et al. COVID-19 lombardy ICU network. baseline characteristics and outcomes of 1591 patients infected with SARS-CoV-2 admitted to ICUs of the Lombardy Region, Italy. JAMA. 2020 Apr 6: e205394. doi:10.1001/jama.2020.5394.

27. Niger reports new polio outbreak. [accessed 2020 Jun 29]. https:// www.who.int/immunization/diseases/measl es/statement_missing_

measles_vaccines_covid-19/en/.

28. Roberts L. Why measles deaths are surging - and coronavirus could make it worse. Nature. 2020 Apr;580(7804):446-47. doi:10.1038/ d41586-020-01011-6.

29. Wadman M. Measles epidemic in Ukraine drove troubling European year. Science. 2019 Feb 15;363(6428):677-78. doi:10.1126/science.363.6428.677.

30. Niger reports new polio outbreak. [accessed 2020 Jun 29]. https:// www.who.int/immunization/diseases/measl es/statement_missing_ measles_vaccines_covid-19/en/.
31. Hoffman J, Maclean $R$ Slowing the Coronavirus is speeding the spread of other diseases. New York Times. 22 Jun 2020. https:// www.nytimes.com/2020/06/14/health/coro navirus-vaccines- measles.html. [accessed 2020 Jun 29]

32. Behrens L, Cherry JD, Heininger U, the Swiss Measles Immune Amnesia Study Group. The susceptibility to other infectious diseases following measles during a three year observation period in Switzerland. Pediatr Infect Dis J. 2020 Feb 14. doi:10.1097/ INF.0000000000002599.

33. Gostin LO, Salmon DA. The dual epidemics of COVID-19 and influenza: vaccine acceptance, coverage, and mandates. JAMA. Jun 112020.

doi:10.1001/jama.2020.10802.

34. Vaccination Guidance During a Pandemic. [accessed 2020 Jun 29]. https://www.cdc.gov/vaccines/pandemic -guidance/index.html.

35. Vaccinations of newborns in the context of the COVID-19 Pandemic. https://iris.paho.org/bitstream/handle/1066 5.2/52226/ PAHOFPLIMCOVID19200010_eng.pdf?sequence= 1 \&isAllowed=y. [accessed 2020 Jun 29]

36. Immunization in the context of COVID-19 pandemic.

https:// www.who.int/publications/i/item/immuniza tion-in-the-context-of -covid-19-pandemic. [accessed 2020 Jun 29]

37. Interim guidance on continuity of immunization programs during the COVID19 pandemic. [accessed 2020 Jun 29]. https://www. canada.ca/en/publichealth/services/immunization/nationaladvisory-committee-on-immunization- 
naci/interim-guidance- immunizationprograms-during-covid-19-pandemic.html.

38. Hotez PJ, Nuzhath T, Colwell B. Combating vaccine hesitancy and other 21 st century social determinants in the global fight against measles. Curr Opin Virol. $2020 \mathrm{Feb}$ 26:41:1-7. doi:10.1016/j. coviro.2020.01.001.

39.Zarocostas J. How to fight an infodemic. Lancet. 2020;395 (10225):676. doi:10.1016/S0140-6736(20)30461-X.

40. Amimo F, Lambert $B$, Magit $A$. What does the COVID-19 pandemic mean for HIV, tuberculosis, and malaria control? Trop Med Health. 2020 May 13:48:32. doi:10.1186/s41 182-020-00219-6.

41. Kluge HHP, Jakab Z, Bartovic J, D'Anna V, Severoni S. Refugee and migrant health in the COVID-19 response. Lancet. 2020 Apr 18;395(10232):1237-39. doi:10.1016/S01406736(20)30791-1.

42. [accessed 2020 Jun

https://en.unesco.org/covid19/

educationresponse.

43. Santoli JM, Lindley MC, DeSilva MB, Kharbanda EO, Daley MF, Galloway L, Gee J, Glover M, Herring B, Kang Y, et al. Effects of the COVID-19 pandemic on routine pediatric vaccine ordering and administration - United States, 2020. MMWR Morb Mortal Wkly Rep. 2020 May 15;69(19):591-93.

doi:10.15585/mmwr.mm6919e2.

44. Netea MG, Giamarellos-Bourboulis EJ, Domínguez-Andrés J, Curtis $N$, van Crevel $\mathrm{R}$, van de Veerdonk FL, Bonten M. Trained immunity: a tool for reducing susceptibility to and the severity of SARS-COV-2 infection. Cell. 2020 May 28;181(5):969-77. doi:10.1016/j.cell.2020.04.042. 